\title{
Option Values, Switches, and Wages: An Analysis of the Employment Guarantee Scheme in India
}

\author{
Pasquale Scandizzo, Raghav Gaiha, and Katsushi Imai*
}

\begin{abstract}
Consistent with real option theory, the authors argue that the value of the Employment Guarantee Scheme (EGS) in rural India and its impact on workers' behavior does not depend so much on its income supplementation as on enlargement of opportunities in the uncertain local labor market. The choice between the EGS and other activities is modeled in a dynamic optimization framework, taking into account a fixed wage rate and certainty of employment under the EGS and a stochastic wage rate under other activities. Specifically, volatility of wages in the rural labor markets has important implications for switches into the EGS and for concomitant welfare effects. Under such conditions, the higher the EGS wage, the greater is its attractiveness to relatively skilled and affluent workers, and for those already in it to continue. These and related predictions of the model are validated by panel data estimation.
\end{abstract}

\section{Introduction}

Much of the earlier literature focuses on the targeting and poverty-alleviating potential of the Employment Guarantee Scheme (EGS) in the Indian state of Maharashtra (e.g. Ravallion, 1991; Ravallion and Datt, 1995; Gaiha, 2000). Attention is given to various mechanisms through which it impacts on the rural poor. These include direct transfer benefits as well as indirect ones through a positive effect on agricultural wage rates. A point of departure of the present study is the focus on switches into the EGS in a framework consistent with the theory of real options. This theory appears relevant for the problem at hand, because the value of the EGS and its impact on workers' behavior do not depend so much on income supplementation as on the enlargement of opportunities that it provides in the uncertain environment of the local labor market, encompassing both farm and non-farm activities. Thus, rather than actual increase in income and employment, the EGS promises potential increases of these variables for given levels of volatility in the regular labor market, or, alternatively, potential decreases in volatility for given levels of income and employment. As in most insurance schemes, these effects, in turn, may change workers' behavior in a way that may not be fully consistent with ex ante conditions. For example, the extent to which workers diversify their portfolio of activities may be reduced and a larger proportion of workers may participate in the regular labor market, rather than in self-employment in farm or non-farm activities, because the EGS provides a form of employment of last resort, at a fixed wage, that can be readily used to cover unemployment and wage risks.

\footnotetext{
* Scandizzo: University of Rome "Tor Vergata," Via Columbia 2, 00136 Rome, Italy. Gaiha: Faculty of Management Studies, University of Delhi, Delhi, 110007, India. Imai: Department of Economics, School of Social Sciences, University of Manchester, Oxford Road, Manchester M13 9PL, UK. Tel: + 44-161-2754827; Fax: + 44-161-275-4928; E-mail: Katsushi.Imai@manchester.ac.uk. We have benefited from comments on the earlier version from participants in meetings at the Royal Economic Society and in seminars at Oxford, Manchester, and Osaka City University. Our discussions with D.M.G. Newbery, C.J. Bliss, and J. Toye clarified a number of points. We are also grateful to the editor and an anonymous reviewer for their meticulous suggestions. We alone are, however, responsible for any remaining deficiencies.
} 
The choice between the EGS and other activities in rural areas-including farm and non-farm employment-is modeled in a dynamic optimization framework that takes into account a fixed wage rate and certainty of employment under the EGS (for a fixed period of time) and a stochastic wage rate under other activities, over different seasons. Allowance is also made for volatility of regular labor market activities-in the present case, agricultural wage earnings. The focus therefore is on switches between these alternatives on the basis of a (discounted) cash flow analysis that encompasses these factors. Some interesting insights into participation in the EGS emerge. The higher the EGS wages, for example, the higher would be the proportion of higher income workers for two mutually reinforcing reasons: higher EGS wages (net of entry costs) will induce higher income workers to opt out of other labor market activities in favor of the EGS; and higher EGS wages would also make fewer higher income participants opt out of this scheme in response to expected wage increases in other labor market activities. Moreover, because higher expected incomes can be used to offset entry and exit costs, the EGS would also tend to select a disproportionately larger share of higher income workers, as they can afford to switch back and forth more easily than less affluent ones. Indeed, some of these insights may throw new light on and explain better the worsening of mistargeting of the EGS during the 1980s (Gaiha, 2000) - a period marked by a sharp rise in EGS wage rates. But, more generally, premised as they are on a given wage rate in regular labor market activities, the screening and deterrent arguments that are invoked to support workfare programs-of which the EGS is a special case-need to be reformulated. ${ }^{1}$ Guided by the option value model developed here, an empirical analysis of switches into the EGS will be carried out.

The scheme is as follows. In section 2, salient features of the EGS are described briefly. This is followed by a short description of the ICRISAT panel survey in section 3. Section 4 contains a condensed (non-mathematical) version of the option value model of participation in different activities, including the EGS, with some elaboration of the technical details given in the Appendix. In Section 5, an econometric analysis of switching is provided. The paper concludes with a brief review of the main findings from a broad policy perspective.

\section{EGS: Salient Features ${ }^{2}$}

The EGS has served as the most important poverty alleviation program in Maharashtra since the late 1990s. The scheme guarantees that every adult who wants a job in a rural area will be given one, provided that the person is willing to do unskilled manual work on a piece-rate basis. Self-selection of the poor is built into the EGS. First, no choice of work is offered. Second, until 1988, the wage rate was usually below the agricultural wage rate. ${ }^{3}$ Third, as the guarantee holds at the district level, a person may be required to travel a long distance for a few days of temporary work.

The employment seeker is required to work for a minimum of 30 days on the site assigned. The person must present him or herself for work within seven days of the offer. Failure to provide employment within 15 days of registration entitles the person to an unemployment allowance (of Rs. 2 per day). ${ }^{4}$ Despite the decline of participation in the 1980s and 1990s, the EGS continues to perform an important role of stabilizing income in backward regions (Gaiha, 2005).

Our analysis has important implications for the National Rural Employment Guarantee (NREG) program which was launched in February, 2006. Initially, it was confined to the most backward 150 districts, extended later to a further 330 districts, and 
recently to all districts. Some features of the NREG are controversial. These include adherence to the statutory minimum wage and vesting of responsibility for implementation in village panchayats without required capacity building (Gaiha, 2003; Dreze, 2004; Murgai and Ravallion, 2005). Various assessments of the NREG point to huge leakages and dismal targeting (Biswas, 2007), while Jha et al. (2008) find that the targeting performance of the NREG program is satisfactory based on more recent small-scale surveys in Rajasthan. Our analysis offers the insight that the higher the EGS wage (or, for that matter, the NREG wage), given the volatility of marketdetermined wage rates, the greater is the attractiveness of this scheme to the relatively skilled and affluent workers. Besides, there is a stronger incentive for those who are already in the scheme to remain in it within the prescribed limit of a certain number of days.

\section{ICRISAT Panel Survey ${ }^{5}$}

The empirical part of the present study is based on the ICRISAT panel data from 1975 to 1984 , which cover production, expenditure, time allocation, prices, wages, and socio-economic characteristics for 240 households in six villages, representing three agro-climatic zones in the semi-arid region of south India. Given the agro-climatic conditions and purposive selection of the villages, our data are not representative of all of rural south India or, for that matter, even of its semi-arid region. Nevertheless, the longitudinal nature and richness in terms of variables included are what make the ICRISAT data unique. The present analysis is based on the samples for two villages, Shirapur and Kanzara in Maharashtra, for which the data on participation in the EGS are available.

\section{The Model}

The traditional approach to the analysis of employment programs has considered the benefits of a separating equilibrium, based on the self-selection characteristics of the work-salary combination (Besley and Coate, 1992). In a situation of dynamic uncertainty, however, we may conjecture that self-selection may be less effective, for several reasons. First, a separating equilibrium may not exist, that is, a given salary in the EGS may be equally or even more attractive for higher skilled than for lower skilled workers. The latter may face exit costs from the labor market (for example, loss of reputation as "loyal" workers) that may make their prospective EGS income less attractive than in purely monetary terms. Also, rather than acting on a once-and-for-all allocation of labor, the employment program may motivate workers to switch back and forth between the program and the labor market in response to threats and opportunities provided by stochastic wages. Because higher skilled workers are better off economically and command a higher market power, they may be expected to afford entry and exit costs much more easily than low skilled workers. While its impact is more difficult to hypothesize, uncertainty appears to be an additional, important factor in determining differential behavior of poor and better-off workers. On the one hand, higher wages may be more volatile, thus motivating higher skilled workers to move more frequently from one position to another, in and out of the labor market. On the other hand, lower wages are generally associated with higher risks of unemployment, so that, once a poor worker is out of the labor market, it may be more difficult for him or her to find a job again, even though this would be desirable at the current wages. 
In order to explore these factors and their impact on the EGS, we have resorted to the use of real option theory. This is a recent innovation in decision theory, which promises to be of great relevance in modeling and analyzing all problems where decisions are taken, not only under conditions of imperfect information, but also of dynamic uncertainty. This last term indicates a situation where uncertainty evolves over time, as a consequence of the new information that becomes available as sequences of different states of nature materialize. Unlike traditional decision-making analysis, which concentrates exclusively on expected present values of future benefits and costs, methodologies based on real option theory are based on the idea that the decisionmaker holds one or more "options", i.e. rights, but not obligations, to undertake certain actions whose outcomes are uncertain. As a consequence, these methodologies focus on the risks and opportunities arising from the uncertain unfolding of events over time.

In the case of a rural worker, in particular, the traditional analysis considers his or her decision problem as one of optimal allocation of his or her labor resources, given certain expectations on the levels of variables, such as wage levels and the probability of finding employment, in agricultural and non-agricultural markets. According to real option theory, we consider instead the worker to be endowed with a real option reflecting the working opportunities open to him or her, given his present status. In particular, we consider the case of an agricultural worker who may choose to allocate his or her labor supply between two alternative uses. The worker could find employment in the regular market for a wage whose size depends on market conditions as well as on the probability and the length of employment. Or, he or she may decide to join an employment scheme with a fixed wage and a known portion (possibly all) of his or her employment time. Joining the scheme, however, will entail a cost for three reasons: first, it will cause average employment time in the regular labor market to fall; second, it will involve administrative expenses (red tape) in the form of money and time loss; third, it may cause a loss of reputation of the worker in the labor market.

We assume that participation and exit costs consist of commitments of nonrecoverable resources and that workers decide for every period (say, different seasons) the regime that they will follow, by predicting the discounted cash flow (DCF), possibly adjusted for risk aversion, which they will obtain by choosing one of the employment alternatives. For simplicity, we assume that a (typical) worker evaluates his or her position as if the corresponding income stream were to last indefinitely. ${ }^{6}$ We model the employment in the regular market as a search process, whereby the worker samples jobs for openings and salaries from an underlying random distribution. Within a given interval of time (for example, a season), wage income in the regular market is assumed to change in such a way that the variance of mean income shift, expressed in percentage terms, grows linearly with time, as participation in the labor market increases and laborers confront a higher degree of job and task differentiation. Employment in the regular market is thus assumed to yield a net cash flow $y$, evolving according to a stochastic process of the geometric, Brownian-motion variety with zero drift:

$$
d y=\sigma y d z
$$

where $\sigma^{2}$ is the variance and $d z$ is a normally distributed random variable such that $E d z=0$ and $E d z^{2}=d t$.

The increase in the process volatility with time depends on the characteristics of the random process (the so-called "random walk"), combining single random movements with the passage of time. As a consequence of the latter, the salary level at any point in time will depend on the salary level achieved in the previous period, so that the spread over time of the possible wage values will increase linearly, just as, for example, the 
range of all possible positions of a frog jumping from one place to another increases with the distance of the timing of a single jump from the beginning of the observations. In the case of the labor market, increasing variance over time implies that the worker, even within the same season, faces larger uncertainty if he or she tries to make a longer term prediction (i.e. further from the beginning and closer to the end of the season, or even for later seasons) of the wage level. His or her decision-making problem in choosing the current job or the possible recourse to the employment scheme is thus complicated by the dynamic nature of uncertainty, which becomes more and more pervasive, the longer he or she tries to look into the future.

The option to participate in the public employment scheme can be considered a "put" option, i.e. a faculty, given to the workers employed in the regular labor market, to join the program, should the regular salary or wage (or the probability of employment) fall below a certain critical level. ${ }^{7}$ The value of such an option, $F(y)$, can either be determined by replicating it with a portfolio of assets and liabilities (Dixit and Pindyck, 1994:189) or, more simply, by using dynamic programming. This is a technique that allows us to reduce a maximization problem over time to a single, repetitive choice for each point in time.

We assume that the worker maximizes the expectation of the discounted utility of his or her future incomes and consider the case in which the worker is supposed to change his or her status only once in each season. In this case, which is by far the most common one in practice, we can think of the options available to the worker as triggered by a spline of stochastic processes of the same type as in equation (1), but whose parameters change at each turn of the season. In this case, the worker's problem becomes a sequential one (Knudsen and Scandizzo, 2002).

Given this background, we derive the following propositions: ${ }^{8}$

Proposition 1. The critical level of income from the regular labor market that causes the worker to join the program increases as the program wage increases, as the program entry costs decrease, as the time to the end of the season decreases, and as the level of uncertainty decreases.

PROPOSITION 2. The critical level of income from the regular labor market, at which the worker decides to re-enter the market, increases as the program wage increases, as the re-entry costs increase, as the time to the end of the season decreases, and as the level of uncertainty increases.

Propositions 1 and 2 suggest that there may be several limits to the effectiveness of the EGS as a mechanism to administer selectively a transfer to lower income workers. On the one hand, for any given level of program wages, the fact that effective income in the program depends on the worker's productivity (piecewise performance) will tend to select higher skill and more hard-working laborers. This will induce, ceteris paribus, a larger proportion of higher income workers to leave the labor market for the program. On the other hand, higher program incomes (or, equivalently, wages) will also make fewer higher income people leave the program in response to expected income increases from the labor market. Furthermore, because higher expected incomes can be used to offset entry and exit costs, the program may also select a disproportionately larger share of higher income persons, because they expect to be able to afford to switch back and forth more easily (being able to pay the related costs) than those with low incomes. Also, it is reasonable to hypothesize that higher labor incomes are associated with higher wage volatility, while lower incomes are associated with a higher risk of 
being unemployed. Greater wage uncertainty for higher income workers implies that they will be more inclined, ceteris paribus, to join the program and, once in it, more inclined to stay there. Finally, the uncertainty, and entry and exit costs, will determine a band of inaction that will tend to be different for different workers and may also change over time according to the workers' incomes and skills. As we will see below, the effect of uncertainty is twofold: on the one hand it tends to increase the rate at which workers switch in and out of the program; on the other hand, by increasing the size of the band of inaction, the uncertainty discourages switching for the individual worker.

Proposition 3. Given a distribution of workers by income between a maximum value attainable and zero, the rate at which the workers will exit the labor market to join the EGS increases as the difference between the maximum value of income from the regular labor market and the exit value decreases.

An intuitive interpretation of this result is as follows. Clearly, if all workers were located at the exit value of income (i.e. the difference between maximum and exit income tended to zero), the rate of exit would be the maximum possible. More generally, for a finite difference, the workers who are candidates for switching are distributed between the maximum value of income attainable and the exit value. Consider the workers in the neighborhood of a particular value of income. If the distribution at any income level is stationary, then the workers leaving their location because of income decreases should be exactly counter-balanced by the workers arriving in the location because of income increases. The rate at which workers will exit the labor market to join the EGS is thus the fraction of the workers located just to the right of the exit income. But for any given distribution, this fraction increases as the distance from the upper bound of the range decreases. Therefore, the smaller the gap between any given income and the exit income, the larger is the rate at which workers will exit from that location.

COROLlary. The effect of uncertainty on the rate at which the workers exit the labor market or join the EGS has two components: one positive and the other negative. The negative component increases as the EGS wage rate increases.

Because the rate at which the workers exit the market depends on the density of the workers located just to the right of the exit income, if uncertainty increases, this density will be affected in two different ways. On the one hand, a higher volatility of workers' incomes will increase the number of workers whose incomes may, in some states of the world, be in the critical zone. On the other hand, for any given level of the EGS salary, a larger number of workers will find that it is better to wait than to switch to the program. With higher uncertainty, the value of waiting increases, and the exit rate tends to decrease correspondingly.

Proposition 4. Assume that a given worker faces a distribution of jobs by income between a maximum value attainable and zero. Then, the frequency at which the worker will exit the labor market to join the EGS will increase as the difference between the maximum value of income from the regular labor market and the exit value of income decreases.

This proposition asserts that any worker, confronted with a distribution of jobs with different incomes, is equivalent to a population of workers with a given job. Thus, the 
rate at which a particular worker is going to join the EGS is governed by the same rule that governs the exit rate of a population of workers holding a specific job at any one time. This proposition has a higher relevance for the targeting of the program, however. In fact, it asserts that the number of switches between the program and the regular labor market will tend to be higher for workers whose income above the exit value varies within a smaller range. Thus, workers that expect their salary in the regular market to vary within a smaller range will tend to switch more frequently, ceteris paribus, between the two alternatives.

COROLlary. The effect of uncertainty on the rate at which a given worker will switch from his or her regular job to join the EGS has two components: one positive and the other negative. The negative component increases as the EGS wage rate increases.

The switching frequency at which the worker exits the market to join the EGS depends on the density of jobs located just to the right of the exit income. Thus, if uncertainty increases, this density will be affected in two different ways. On the one hand, a higher volatility of incomes will increase the number of times where income may, in some states of the world, be in the critical zone. On the other hand, if the EGS salary is sufficiently large, compared to their present salary, when the workers finds themselves in a situation where they contemplate quitting their jobs to join this program, they can also afford to wait longer. With higher uncertainty, the value of waiting increases, and the switching rate tends to decrease correspondingly.

In sum, the results obtained for the number of workers switching between the regular labor market and the EGS generalize to the number of switches of an individual worker. The econometric analysis that follows is confined to the latter.

\section{Estimation and Results}

\subsection{Wage Equation}

As the ICRISAT panel survey does not contain estimates of farm or EGS wage rates, we have constructed two alternative sets. In the first set, actual wage earnings in these activities are divided by the number of days worked by each individual in a year and then ratios of wage rates are computed. In the second, ratios of wage rates in the EGS and in farm activities are posited to be endogenous to individual, household, and village characteristics. A random-effects Tobit specification is used to estimate the wage ratio functions, as shown below.

$$
W_{\text {egsitv }} / W_{\text {agritv }}=f_{i t v}\left(H_{i t v}, S_{i t v}, A_{i t v}, B_{i t v}, V_{i t v}, R_{t v}, \alpha_{i v}\right)
$$

As specified in this equation, the ratio of EGS and farm wage rates is determined by a health indicator (i.e. body mass index, BMI) denoted by $H$, a schooling index (number of years) denoted by $S$, a vector of sociodemographic characteristics (e.g. age, gender, caste, whether married) denoted by $A$, other household characteristics (e.g. occupation of household head, household debt) denoted by $B$, a measure of wealth (or land owned) denoted by $V$, a measure of aggregate risk faced by households (i.e. coefficient of variation of monthly rainfall) denoted by $R$, an unobserved factor (say, ability) denoted by $\alpha$, and $i$ indexes the individual, $t$ represents the year $(t=1$ for $1979, \ldots, t=6$ for 1984), and $v$ denotes the village.

Table 1 contains the results of the determinants of the ratio of EGS and agricultural wage rates. While the BMI and its square do not influence this ratio, age has a negative 
Table 1. The Random-effects Tobit Estimation Result of the Ratios of Wage Rates (Dependent Variable: [EGS Wage] / [Daily Agricultural Wage])

\section{Constant}

H_BMI (body mass index)

$-0.35(-0.68)$

H_BMI squared (square of body mass index)

$0.006(1.11)$

$A_{-}$age (years) (age of household head)

$A_{-}$female (whether household head is female)

$-0.00016(-1.11)$

$A_{-}$high caste (whether in high caste)

$-0.002(-1.72)^{*}$

$-0.89(-2.63) * * *$

$A_{-}$medium high caste (whether in medium high caste)

$0.02(0.35)$

$0.03(0.58)$

$A_{-}$medium low caste (whether in medium low caste)

$0.11(1.96)^{* * *}$

$S$ (years of education)

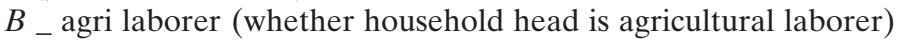

$-0.0092(-1.72)^{*}$

$0.17(3.86)^{* * * *}$

$V_{-}$land (land owned: acres)

$-0.002(-1.46)$

$I_{-}$Shirapur (whether from Shirapur)

$-0.04(-1.07)$

$R$ _cv rain (The coefficient variation of rainfall)

$-0.00008(-0.10)$

$R$ _unemp (-1) (The first lagged unemployment rate)

Number of observations

Joint significance (Wald chi-square)

$\chi^{2}(13)=59.39 * * *$

* Significant at $10 \%$; * significant at $5 \%$; ** significant at $1 \%$.

effect. This is plausible given that EGS wage rates are piece rates calculated on the basis of work done. The female dummy has a negative coefficient, implying much lower wages for female participants in the EGS. Relative to the lowest caste individuals, the medium low caste individuals have higher wage ratios, presumably as a result of higher EGS wage rates. Schooling has a negative coefficient, presumably as a result of a positive effect on agricultural wage rates. Individuals belonging to agricultural labor households also have higher wage ratios-especially because of higher EGS wage rates. The lagged unemployment rate has a positive coefficient, implying a stronger dampening effect of unemployment on farm wage rates as a result of a lowering of reservation wage rates. The overall specification is validated by a Wald test.

\subsection{Switching Equations}

The number of switches between different activities over the entire sample period of six years (1979-84) is calculated as follows: total number of switches of all individuals in the workforce aggregated over six years / total number of individuals in the workforce $\times 6$. Thus the switches denote average switches of a worker in a year. A striking feature is that generally switches into and out of the EGS are relatively high. So, for validation of our option value model, we shall concentrate on the switches into the EGS, which is denoted by $e$.

$$
e_{i t}=e_{i t}\left(W_{\text {egsit }} / W_{\text {agrit }}, L_{i t-1}, H_{i t}, S_{i t}, A_{i t}, B_{i t}\right)
$$

This equation is estimated as Poisson regressions, as the sample size is large and the number of switches is small (more specifically, because of a preponderance of zeros and small positive values). The results are given in Table 2 .

Firstly, in the absence of direct estimates of wage rates, two ratios are used: those based on actual earnings in these activities, and estimated values. Some results are 
Table 2. Poisson Regression Analysis of Switches into the EGS (Dependent Variable: Number of Switches)

\begin{tabular}{|c|c|c|}
\hline & $\begin{array}{c}\text { Case 1: Based } \\
\text { on the actual } \\
\text { wage ratio }\end{array}$ & $\begin{array}{c}\text { Case 2: Based } \\
\text { on the predicted } \\
\text { wage ratio }\end{array}$ \\
\hline Explanatory variables & Coefficient ( $z$ value) & Coefficient ( $z$ value) \\
\hline Constant & $3.23(0.69)$ & $2.07(0.37)$ \\
\hline $\begin{array}{l}\text { EGS participation dummy }(-1)^{1} \text { (whether } \\
\text { participates in the EGS in the previous year) }\end{array}$ & $0.37(1.66)^{*}$ & $0.61(2.79)^{* * *}$ \\
\hline $\begin{array}{l}\text { The actual wage ratio: [EGS wage } \\
\text { rate]/[agricultural wage rate] }\end{array}$ & $1.82(8.92)^{* * *}$ & \\
\hline $\begin{array}{l}\text { The predicted wage ratio: [EGS wage } \\
\text { rate]/[agricultural wage rate] }\end{array}$ & & $3.19(1.97)^{* *}$ \\
\hline H_BMI (body mass index) & $0.35(-0.76)$ & $-0.28(-0.48)$ \\
\hline$H_{-} \mathrm{BMI}^{1}$ (square of body mass index) & $0.0079(0.67)$ & $0.0073(0.49)$ \\
\hline$S$ (years of education) & $-0.05(-1.19)$ & $-0.05(-1.09)$ \\
\hline$A_{-}$female (whether female or not) & $-0.27(-0.47)$ & $-0.68(-1.15)$ \\
\hline$A_{-}$age (years) & $-0.02(-1.55)$ & $-0.01(-1.27)$ \\
\hline$A_{-}$high caste ${ }^{1}$ & $-0.14(-0.38)$ & $-0.22(-0.60)$ \\
\hline$A_{-}$medium high caste ${ }^{1}$ & $0.39(0.98)$ & $-0.06(-0.14)$ \\
\hline$A_{-}$medium low caste ${ }^{1}$ & $0.12(0.24)$ & $-0.09(-0.18)$ \\
\hline $\begin{array}{l}A_{-} \mathrm{CV} \text { (coefficient of variation) in agricultural } \\
\text { wage for each year }\end{array}$ & $-0.007(-3.03)^{* * *}$ & $-0.010(-5.24)^{* * *}$ \\
\hline $\begin{array}{l}A_{-} \text {whether the lagged agricultural wage is in } \\
\text { top } 5 \%\end{array}$ & $0.75(1.98)^{* *}$ & $0.59(1.51)$ \\
\hline $\begin{array}{l}B_{-} \text {agri labourer }{ }^{1} \text { (whether household head is } \\
\text { agricultural laborer) }\end{array}$ & $0.03(0.13)$ & $-0.75(-1.73) *$ \\
\hline$I_{-}$Shirapur (whether from Shirapur) $)^{1}$ & $0.41(1.23)$ & $0.29(0.87)$ \\
\hline$A_{-}$female* $A_{-}$high caste & $-0.50(-0.66)$ & $0.39(0.52)$ \\
\hline$A_{-}$female* $A_{-}$medium high caste & $-0.48(-0.72)$ & $0.39(0.52)$ \\
\hline$A_{-}$female* $A_{-}$medium low caste & $-0.48(-0.66)$ & $-0.46(-0.66)$ \\
\hline$A_{-}$female* $I_{-}$Shirapur & $0.49(0.81)$ & $0.79(1.40)$ \\
\hline Year dummy (year 1980) & $-1.04(-1.71) *$ & $0.29(0.65)$ \\
\hline Year dummy (year 1981) & $-0.99(-1.56)$ & \\
\hline Year dummy (year 1982) & $0.16(-0.28)$ & $0.68(1.66)^{*}$ \\
\hline Year dummy (year 1983) & $-0.78(-1.32)$ & $0.29(0.62)$ \\
\hline Year dummy (year 1984) & $-0.86(-1.48)$ & $0.70(1.51)$ \\
\hline Number of observations & 367 & 356 \\
\hline Joint significance $\left(\mathrm{LR} \chi^{2}\right)$ test & $\chi^{2}(23)=212.03 * *$ & $\chi^{2}(22)=116.34 * *$ \\
\hline Goodness-of-fit $\left(\chi^{2}\right)$ test & $\chi^{2}(343)=151.44 * *$ & $\chi^{2}(333)=232.29 * *$ \\
\hline
\end{tabular}

${ }^{1}$ Dummy variable. * Significant at $10 \% ; * *$ significant at $5 \% ; * * *$ significant at $1 \%$.

similar. In both cases, as predicted by the model, the switches into the EGS are greater, the higher is the ratio of EGS wage rate to farm wage rate. Secondly, the switches into the EGS are also greater among those who participated in this scheme earlier, presumably as a result of an inverse relationship between experience and lower entry costs. Thirdly, the higher the coefficient of variation of agricultural wages, the lower the switches into the EGS. Fourthly, the positive coefficient of agricultural wages being in the top $5 \%$ implies that those in this interval switch into the EGS more often. This 
coefficient is, however, significant only in the case of the actual wage ratio. With the actual wage ratio, the dummy for 1981 has a negative coefficient, implying lower switches in this year. In the predicted wage ratio case, on the other hand, the presence of a household head who is an agricultural laborer has a negative coefficient, implying lower switches into the EGS. Because agricultural labor households are highly poverty prone or mostly at the lowest tail of income distribution, the lower number of switches among them could well be due to high entry costs or negative reputation effects (given the nature of casual labor contracts in rural India). Also, the coefficient of the dummy for 1982 has a positive coefficient, implying more switches into the EGS in this year. The goodness-of-fit test does not reject the Poisson functional form and the joint significance test confirms the overall specification of this regression.

What then are the implications of these results? The first important point is that switches into the EGS are governed by the (corresponding) wage ratio. The higher the EGS/farm wage ratio, the greater are the switches into the EGS, in accordance with a higher discounted cash flow. The wage ratios do not, however, reflect fully the entry and exit costs. It should be noted, as the second important point, that the switches are greater among those who worked in the same activity in the previous year. To the extent that this reflects lower entry costs into the EGS (e.g. due to greater familiarity with registration procedure), subject to the caveat noted earlier, this further corroborates the central argument of the option value model. Higher switches among a top subset of agricultural wage earners (i.e. the top 5\%) lend additional support to this argument. A third important point is that greater volatility of agricultural wages has a dampening effect on switches into the EGS, consistent with a larger band of inertia from the previous activity or agriculture.

There was a worsening of the targeting of the EGS over the period 1979-89. During 1988, there was a sharp upward hike in the EGS wage rate. In fact, over the period 1983-91, with one exception, the EGS wage rate exceeded the farm wage rate and the gap widened sharply in 1989. As shown in Gaiha (2000), the targeting of the EGS also worsened with a much higher share of participants belonging to relatively affluent households.

To further explore the reasons underlying the mistargeting of the EGS, we examined the relationship between switches into the EGS and the EGS wage rates. Because the latter are determined on a piece-rate basis, the higher skilled and more hardworking individuals would tend to switch into the EGS and earn higher wages, given slackseason options in farm and non-farm activities. Moreover, considering the band of inertia, they are more likely to remain in the EGS. That the switches into the EGS are in fact greater among those earning higher EGS wages is confirmed by our analysis of the ICRISAT data. What is perhaps equally significant is the confirmation that both EGS and agricultural wage rates are higher depending on land owned. Thus the mistargeting of the EGS was in part a reflection of greater switches of the higher skilled and wealthier workers, induced by the prospect of more remunerative opportunities in this scheme.

What the preceding analysis suggests is that, while the wage ratio matters, this by itself cannot explain the worsening of the targeting. The choice between the EGS and regular labor market activities (in the present context, agriculture) must take into account discounted cash flows associated with them, the entry and exit costs, and the volatility of agricultural wage rates. The option value model used here demonstrates how these considerations could be incorporated in a dynamic optimization framework. In particular, what emerges from this analysis is that the case for rural public works is a more complex one, as it must go beyond the screening and deterrent arguments 
premised on a fixed wage differential. Specifically, volatility of market-determined wage rates in rural areas is key to understanding the choice between the EGS and other employment options in rural areas.

\section{Concluding Observations}

Some observations are made here to put the main findings in perspective. A point of departure of this analysis is the focus on the switches that occur between the EGS-a rural public works scheme with guaranteed employment at a fixed wage rate-and regular labor market activities with a market-determined stochastic wage rate. As a result, the emphasis is on employment options judged on the basis of their discounted cash flows. So not just the wages in different activities but also entry and exit costs matter in labor supply decisions in the option value framework. The econometric evidence confirms that switches into the EGS are positively linked to the EGS/farm wage ratio. It also suggests that switches are linked to variables that proxy some of the entry and exit costs (e.g. employment in the EGS in the previous year). Further, there is evidence confirming greater participation of a top subset of agricultural wage earners in the EGS, reflecting their ability to afford better the higher entry and exit costs. But, more importantly, volatility of agricultural wage rates matters a great deal. The higher the EGS wage rate, the greater is the incentive for relatively affluent and skilled workers to participate in it. Also, the greater is the incentive to those who are already in it to continue (or, given the EGS wage rate, the greater the volatility of market wage rates, the higher would be the participation of more skilled and relatively affluent workers). Taking some of these factors into account in a dynamic optimization framework, the analysis suggests that the selective power of the EGS may be impaired by several biases in favor of higher income individuals and by the fact that program entry and exit are not determined by a single income level but by bands of inertia of different size. An explanation based on these factors may help us to understand better the worsening of the targeting of the EGS during the 1980s, as well as the dismal targeting of the NREG program. Because switches into the EGS and regular labor market activities are a result of the joint influence of all these factors, through changes in economic incentives in a volatile rural labor market, there is a risk of overstating the dependence of the participants - especially low income households-on the former.

In conclusion, the analysis conducted suggests that the incentive case for rural public works schemes such as the EGS, in terms of screening and deterrent arguments, appears much less compelling once it is reformulated in a dynamic optimization framework, taking into account discounted cash flows, entry and exit costs, and volatility of agricultural wage rates. If an allowance is also made for the bands of inertia, dependent on incomes and individual levels of uncertainty, the incentive case for workfare is further weakened.

\section{Appendix: Mathematical Proofs}

We assume that a stochastic process of the geometric Brownian-motion variety, with zero drift, governs the worker's income in the regular labor market:

$$
d y=\sigma y d z
$$

The basic criterion of dynamic programming, the so called Bellman equation, prescribes that, in order to maximize a given function over time, the following condition should hold: 


$$
\rho F(y)=E d F(y)
$$

where $\rho$ is an appropriate rate of discount reflecting the worker's opportunity cost for delaying consumption as well as his or her degree of risk aversion.

Equation (A2) states that, in order to maximize the present value of the option, the worker is to equate, in the continuation region (that is, at the margin between holding and exercising the option), the value that he or she would obtain by exercising the option to the expected present value of the future capital gains obtained by holding the option. This condition corresponds to the intuitive optimizing principle that an asset should be disposed of only when the value expected by foregoing it is at least as large as the return from holding it. The dynamic programming technique can thus be viewed as essentially one of continually checking the convenience of continuing a given action (the so called "continuance region") as opposed to abandoning it in favor of its best alternative. Equation (A2) may be used to determine the functional form of the option value function. After applying Ito's lemma, in fact, it yields a partial differential equation whose general solution is:

$$
F(y)=A_{1} y^{\beta 1}+A_{2} y^{\beta 2}
$$

where $A_{1}$ and $A_{2}$ are constants determined by boundary conditions and $\beta_{1}$ and $\beta_{2}$ are, respectively, the positive and the negative root of the characteristic equation:

$$
\rho-\frac{\beta}{2}(\beta-1) \sigma^{2}=0
$$

Proof of proposition 1. We assume that each worker maximizes the discounted value of his or her expected utility of future income:

$$
E \int_{0}^{T} e^{-\rho s} U(y(s)) d s
$$

where $U(y)$ is a well-behaved utility function increasing in income. Because the worker's choice concerns the optimal combination of risky assets, we can apply the Tobin separation theorem, whereby the optimal combination of risky assets for an investor can be determined without any knowledge of the investor's preferences toward risk and return, while preferences are relevant only for establishing the combination of the risky portfolio with the risk-free asset. Thus, the worker can be seen as an investor who has to choose between two risky assets (market or EGS employment with related options). As a consequence, assuming that risks and opportunities of the two alternative forms of employment are captured by the options to enter and exit the program, workers will choose the alternative with the highest expected return. Thus, exit from the program and entry into the program have to meet the two "value matching" conditions (Dixit and Pindyck, 1994:218):

$$
\begin{aligned}
& \int_{t}^{t_{\tau}} y_{e \tau} e^{-\rho s} d s+\sum_{i=1}^{n} A_{1 \tau+i} y_{\tau+i}{ }^{\beta_{1 \tau+i}}+A_{2 \tau} y_{e \tau}{ }^{-\beta_{2 \tau}}=\frac{w}{\rho}-C+\sum_{i=1}^{n} A_{1 \tau+i} y_{\tau+i}{ }^{\beta_{1 \tau+i}} \\
& \frac{w}{\rho}+A_{1 \tau+1} y_{u \tau+1}{ }^{\beta_{1 \tau+1}}+\sum_{i=2}^{n} A_{1 \tau+i} y_{\tau+i}{ }^{\beta_{1 \tau+i}}=\int_{t_{\tau}+t}^{T} y_{u \tau+1} e^{-\rho s} d s-E+\sum_{i=2}^{n} A_{1 \tau+i} y_{\tau+i}{ }^{\beta_{1 \tau+i}}
\end{aligned}
$$


where $\tau$ indicates the low season, with duration from 0 to $t_{\tau}$, and $\tau+1$ the high season, with duration from $t_{\tau+1}$ to $T$, and the subscripts $e$ and $u$ denoting, respectively, entry and exit levels from the program.

Expression (A6) states that at any time $t$ that the worker decides to join the program, his or her expected income from remaining in the (slack season) labor market, plus the value of the option to join the program at any time in the future within the slack season, plus the value of the options to enter the market in any of the next seasons, must equal the expected income from the program over the same time span, minus entry costs, plus the value of the option to go back to the market in the busy season. Because the option to enter the labor market in any of the future seasons is common to both conditions (i.e. being in the market and being in the program), the corresponding term drops out, so that equation (A4) depends only on the option to join the program.

Equation (A7), on the other hand, states that, during the busy season, at any time $t_{\tau+t}$ the worker may switch from the program back to the labor market. At the time of the switch, the discounted value of his or her income from the program over the remaining part of the season, plus the value of the options to re-enter the labor market in the same season, or in one of the future ones, must equal the expected value of his or her income from the market, minus re-entry costs, plus the value of the option to re-enter the market in the next season or in any of the future ones.

In addition to the condition of indifference between the two alternatives in terms of expected incomes (the "value matching condition" in (A6)), in order to achieve optimality, the worker will also have to be indifferent to any marginal increase of expected income and the corresponding increase of its option value, i.e. of the value of the option to switch to the public program. This additional condition (which is referred to as "the smooth pasting condition") is required to ensure that the worker is not only indifferent to the net expected benefits from either alternative (the labor market and the program), but also to the marginal net expected benefits, so that his or her position cannot be further improved by waiting.

Differentiating the value-matching condition in (A6) w.r.t. $y_{t}$, for $y_{t}=y_{e \tau}$, yields:

$$
\int_{t}^{t_{\tau}} e^{-\rho s} d s-A_{2 \tau} y_{e \tau}{ }^{-\left(1+\beta_{2 \tau}\right)}=0
$$

Eliminating the constant $A$ between (A6) and (A8), we obtain the expression for the optimal switching value of labor income:

$$
\frac{y_{e \tau}}{\rho}=\frac{\beta_{2 \tau}}{\beta_{2 \tau}+1}\left(\frac{w}{\rho}-C\right)\left(\frac{1}{e^{-\rho t}-e^{-\rho t_{\tau}}}\right)
$$

Differentiating w.r.t. to $\sigma^{2}$ we find:

$$
\frac{\partial}{\partial \sigma^{2}}\left(\frac{y_{e \tau}}{\rho}\right)=\frac{1}{\left(\beta_{2 \tau}+1\right)^{2}}\left(\frac{w}{\rho}-C\right)\left(\frac{1}{e^{-\rho t}-e^{-\rho t_{\tau}}}\right) \frac{\partial \beta_{2 \tau}}{\partial \sigma^{2}}
$$

which is less than zero because $\frac{\partial \beta_{2 \tau}}{\partial \sigma^{2}} \leq 0$ from (A6).

Similarly, if we consider the condition to exit the program (i.e. equation A8), applying again the smooth-pasting condition and substituting into (A8):

$$
\frac{y_{u \tau+1}}{\rho}=\frac{\beta_{1 \tau+1}}{\beta_{1 \tau+1}-1}\left(\frac{w}{\rho}+E\right)\left(\frac{1}{e^{-\rho t_{\tau+1}}-e^{-\rho T}}\right)
$$




$$
\frac{\partial}{\partial \sigma^{2}}\left(\frac{y_{u \tau+1}}{\rho}\right)=\frac{1}{\left(\beta_{2 \tau}+1\right)^{2}}\left(\frac{w}{\rho}+E\right)\left(\frac{1}{e^{-\rho t+1}-e^{-\rho T_{\tau}}}\right) \frac{\partial \beta_{2 \tau}}{\partial \sigma^{2}}
$$

which is again less than zero because $\frac{\partial \beta_{2 \tau}}{\partial \sigma^{2}} \leq 0$ from (A6).

Proof of proposition 3. We follow the method used by Dixit and Pindyck (1994:272-7) to compute the rate of entry in an industry with stochastic uncertainty. Start with the regular labor market and consider a first group of workers who decide to join the public program. Let $g(y)$ be the density function of such an initial draw of $y$ and $G(y)$ the corresponding distribution function. Of the workers in the labor market, for a number $N\left(1-G\left(y_{e}\right)\right)$ there is a fall in salary that justifies immediate switch to the program. The rest remain in the formal labor market, waiting for a possible fall in salary that may induce them to switch.

The workers who are candidates for switching are distributed between the maximum value $y^{M}$ and the exit value $y_{e}$. Let $N \varphi(y)$ denote the density of these workers at location $y$ and let $d h=\frac{\sigma}{\sqrt{d t}}$ be a small variation of salary. For the density to be stable over time, we must have:

$$
N \varphi(y) d h=N g(y) d h+p N \varphi(y-d h) d h+(1-p) N \varphi(y+d h)
$$

where $p=\frac{1}{2}\left(1+\frac{\sqrt{d t}}{\sigma}\right)$. Expression (A13) states the condition of stationarity of the distribution at any salary level as requiring that the workers leaving (to the left) the location because of salary decreases be exactly counterbalanced by workers arriving in the location because of salary increases. Eliminating the common terms and, using a Taylor expansion, expression (A13) becomes a differential equation:

$$
\frac{1}{2} \sigma^{2} \varphi^{\prime \prime}(y)-\varphi^{\prime}(y)+g(y)=0
$$

whose general solution has the form:

$$
\varphi(y)=C_{1} \exp \left(\gamma_{1} y\right)+C_{2}\left(-\gamma_{2} y\right)+\varphi_{0}(y)
$$

where $\gamma_{1}$ and $-\gamma_{2}$ are the roots of the quadratic expression:

$$
\frac{1}{2} \sigma^{2} \gamma^{2}-\gamma=0
$$

while $\varphi_{0}(y)$ is a specific solution of the differential equation in (A14). Because the expression in the first root increases with $y$, it cannot be associated with an equation describing the exit from the labor market to the government program. The first term on the right-hand side of (A13) must thus be zero.

Assume now that $g(y)=\exp \left(y-y_{M}\right)$, i.e. the initial draw of $y$ is a density of the exponential type. It can be easily verified that a specific solution $\varphi_{0}(y)$ is given by:

$$
\varphi_{0}(y)=\exp \left(y-y_{M}\right) /\left(1-\frac{1}{2} \sigma^{2}\right)
$$

which is non-negative if $\sigma^{2} \leq 2$. Consider now the segment immediately to the right of $y_{e}$. The stability of the distribution over time requires that:

$$
N \varphi(y) d h=N g(y) d h+N \varphi(y+d h)
$$

because the left-hand part is missing due to the exit from the EGS program. Letting $d h$ go to zero, we obtain: $\varphi\left(y_{e}\right)=0$. 
Using this result, we can find a value for the constant $\mathrm{C}_{2}$ and a general solution for expression (A11):

$$
\varphi(y)=-\frac{\exp \left[y_{e}(1+\gamma)-\gamma y-y_{M}\right]}{\left(1-\frac{1}{2} \sigma^{2}\right)}+\frac{\exp \left[y-y_{M}\right]}{\left(1-\frac{1}{2} \sigma^{2}\right)}
$$

The rate at which workers will exit the labor market to join the EGS is the fraction $(1-p)$ of the $N \varphi(y+d h) d h$ workers located just to the right of $y_{e}$. Using a Taylor expansion:

$$
\begin{aligned}
& \frac{1}{2} N\left[\varphi^{\prime}\left(y_{e}\right) d h-\varphi\left(y_{e}\right)\right] d h=\frac{1}{2} N \varphi^{\prime}\left(y_{e}\right)(d h)^{2}=\frac{1}{2} \varphi^{\prime}\left(y_{e}\right) \sigma^{2} d t \\
& \text { i.e. } S=\frac{1}{2} N \sigma^{2} \varphi^{\prime}\left(y_{e}\right)=\frac{1}{2} N \sigma^{2} \frac{-\exp \left[y_{M}-y_{e}\right]}{\left(1-\frac{1}{2} \sigma^{2}\right)}(1+\gamma)
\end{aligned}
$$

Proof of the corollary. Differentiating (A21) with respect to $\sigma^{2}$, we obtain:

$$
\begin{aligned}
\frac{d S}{d\left(\sigma^{2}\right)}= & \frac{1}{2} N \frac{-\exp \left[y_{M}-y_{e}\right]}{\left(1-\frac{1}{2} \sigma^{2}\right)} \\
& \times\left\{\frac{1}{\left(1-\frac{1}{2} \sigma^{2}\right)}-\left(\frac{w}{\rho}-C\right)\left(\frac{1}{e^{-\rho t}-e^{-\rho t_{\tau}}}\right)\left(\frac{1}{\left(\beta_{2 \tau}+1\right)^{2}}\right)\left(\frac{1}{4}+\frac{2 \rho}{\sigma^{2}}\right)\right\}
\end{aligned}
$$

\section{References}

Besley, T. and S. Coate, "Workfare versus Welfare: Incentive Arguments for Work-Requirements in Poverty Alleviation Programmes," American Economic Review 82 March (1992):249-61.

Biswas, S., "India's Job Scheme Disappointing," BBC News Delhi, September 26 (2007). Available at http://news.bbc.co.uk/2/hi/south_asia/7005985.stm.

Dixit, A.K. and R.S. Pindyck, Investment under Uncertainty, Chichester: Princeton University Press (1994).

Dreze, J., "Employment as a Social Responsibility," The Hindu, November 22 (2004).

Gaiha, R., "On the Targeting of the Employment Guarantee Scheme in the Indian State of Maharashtra," Economics of Planning 33 October (2000):203-19.

_ , "Decentralisation and Poverty Reduction," in E. Pernia and A. Deolalikar (eds), Poverty, Growth and Institutions in Developing Asia, London: Palgrave Macmillan (2003).

— Recent Evidence," Asian Survey 45 November/December (2005):949-69.

Government of Maharashtra (GOM), "Employment Guarantee Scheme," MS, Mumbai: Planning Department (1997).

Hirway, I. and P. Terhal, Towards Employment Guarantee in India, New Delhi: Sage Publications (1994).

Jha, R., R. Gaiha, and S. Shankar, "Reviewing the National Rural Employment Guarantee Programme," Economic \& Political Weekly, March 15 (2008):44-8.

Knudsen, O.K. and P.L. Scandizzo, "An Options Approach to Sustainable Development," working paper, World Bank and University of Rome (2002).

Murgai, R. and M. Ravallion, "Is a Guaranteed Living Wage a Good Anti-Poverty Policy?" World Bank Policy Research working paper 3640, Washington DC (2005). 
Ravallion, M., "Market Responses to Anti-Hunger Policies: Wages, Prices and Employment," in J. Dreze and A. Sen (eds), The Political Economy of Hunger, vol. II, Oxford: Clarendon Press (1991).

Ravallion, M. and G. Datt, "Is Targeting through a Work-Requirement Efficient?" in D. van de Walle and K. Nead (eds) Public Spending and the Poor, Baltimore: Johns Hopkins University Press (1995).

Walker, T.S. and J.G. Ryan, Village and Household Economies in India's Tropics, Baltimore: Johns Hopkins University Press (1990).

\section{Notes}

1. See Besley and Coate (1992) for the rationale of workfare programs, such as screening and deterrent arguments.

2. This draws upon GOM (1997).

3. Following a High Court directive, the EGS wage rate was hiked in conformity with the Minimum Wages Act. The piece-rates for different types of manual/unskilled work are so fixed that an average person working diligently for 7 hours a day would earn a wage equal to the minimum wage prescribed for agricultural labor for the concerned zone, under the Minimum Wages Act (GOM, 1997).

4. Hirway and Terhal (1994) draw attention to the use of an elaborate procedure which involves completing several documents, contacting several different persons, and exasperating bureaucratic negligence and bribery.

5. See Walker and Ryan (1990) for the details of ICRISAT survey data.

6. It may be assumed that the worker's expected net present value is calculated with reference to a given predicted duration for the job (for example, up to the end of the season), but this would not add anything to the analysis and would make the notation more cumbersome.

7. More generally, we can assume that the stochastic process $y$ concerns the income obtained by the worker by dividing his or her labor between farm and non-farm activities. Note also that regular market salary is used synonymously with wages.

8. Mathematical proofs of these propositions are given in the Appendix. 